\title{
Parenchymal Brain Laceration as a Predictor of Abusive Head Trauma
}

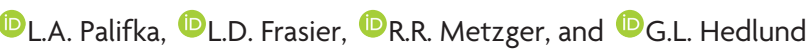

\begin{abstract}
BACKGROUND AND PURPOSE: Accurate differentiation of abusive head trauma and accidental head injury in infants and young children is critical and impacts clinical care, patient prognosis, forensic investigations, and medicolegal proceedings. No specific finding seen on cross-sectional brain imaging has been reported to distinguish abusive head trauma from accidental injury. Our study investigated whether a specific imaging finding, parenchymal brain laceration, is unique to children diagnosed with abusive head trauma.
\end{abstract}

MATERIALS AND METHODS: We retrospectively identified 137 patients with abusive head trauma and 28 patients who incurred moderate to severe accidental brain injury. Brain MR imaging represented the imaging standard for characterizing intracranial injuries.

RESULTS: Among the abusive head trauma cohort, parenchymal brain lacerations were identified in 18 patients, while none were identified in any patients with accidental injury.

CONCLUSIONS: Our findings are in concurrence with the existing forensic, pathology, and imaging literature, which suggests that parenchymal brain lacerations may be related to abusive injury mechanisms.

ABBREVIATIONS: $\mathrm{AHT}=$ abusive head trauma; $\mathrm{Al}=$ accidental injury; $\mathrm{GRE}=$ gradient-echo imaging; $\mathrm{SDH}=$ subdural hematoma; SCWM $=$ subcortical white matter

A busive head trauma (AHT) is an important cause of neurologic morbidity and mortality in children, being most common in infants younger than 1 year of age. Moreover, the fatal consequences and long-term sequelae of AHT are mainly related to primary and secondary parenchymal brain injury, including contusions, axonal shear injury, lacerations, cerebral edema, hypoxia, ischemia, and infarction.

Early signs and symptoms of AHT in infants may be nonspecific, such as irritability, lethargy, or vomiting. Without a history of trauma, these infants are often diagnosed with other conditions that cause similar symptoms in infants. With AHT, historical in-

Received April 13, 2015; accepted after revision June 4.

From the Department of Radiology (L.A.P.), Dartmouth-Hitchcock Medical Center, Lebanon, New Hampshire; Division of Child Abuse Pediatrics (L.D.F.), Penn State Milton S. Hershey Children's Hospital, Hershey, Pennsylvania; Division of Pediatric Surgery (R.R.M.), University of Utah, Salt Lake City, Utah; and Department of Medical Imaging (G.L.H.), Primary Children's Medical Center, Salt Lake City, Utah.

Paper previously presented in part at: Annual Meeting of the American Society of Neuroradiology and the Foundation of the ASNR Symposium, May 17-22, 2014; Montreal, Quebec, Canada.

Please address correspondence to L. Palifka, MD, Department of Radiology, Dartmouth-Hitchcock Medical Center, One Medical Center Dr, Lebanon, NH 03756;

e-mail: Ipalifka@hitchock.org

三 Indicates article with supplemental on-line table.

http://dx.doi.org/10.3174/ajnr.A4519 formation regarding the cause of injury may be difficult to obtain or falsely attributed to an accidental cause, with as many as $30 \%$ of AHT cases being unrecognized as inflicted injury. ${ }^{1}$ Many of those children, if not accurately diagnosed, present later with more serious or even fatal brain injuries.

The clinical and imaging features of inflicted injury, such as retinal hemorrhages, subdural hematoma $(\mathrm{SDH})$, and brain injury resulting in encephalopathy, are highly suggestive of AHT, warranting a comprehensive evaluation for abuse, though they are neither pathognomonic nor always present. ${ }^{2}$ Each case is complex and may have varying features of abusive injury. Therefore, characterizing injuries that are predictive of AHT provides critical information to child abuse specialists who are responsible for the diagnosis and management of AHT. An intracranial finding observed only in AHT, which is lacking in children with witnessed accidental injury (AI), has considerable medicolegal implications. In this observational report, the authors describe a distinct form of inflicted brain injury, parenchymal lacerations, also referred to as subcortical clefts, contusional tears, cerebral contusional white matter clefts, and gliding contusions. ${ }^{1-13}$

Parenchymal brain lacerations have been reported in AHT in conjunction with many of the more specific findings of this condition; however, it is not known at what frequency they occur, if at all, in severe accidental head trauma. Since the initial descriptions 
Table 1: Age and sex of AHT and Al cohorts

\begin{tabular}{lccc}
\hline & $\begin{array}{c}\text { Abusive Head Trauma } \\
(\boldsymbol{n}=137)\end{array}$ & $\begin{array}{c}\text { Accidental Injury } \\
(\boldsymbol{n}=\mathbf{2 8})\end{array}$ & $\boldsymbol{P}$ Value \\
\hline Age, y (median $[\mathrm{IQR}]$, range) & $0.50(0.24-0.92), 0.05-2.92$ & $1.37(0.83-2.38), 0.04-2.83$ & $<.001$ \\
Male & $64 \%$ & $68 \%$ & $\mathrm{NS}$ \\
\hline
\end{tabular}

Note:-NS indicates not significant; IQR, interquartile range.

of traumatic lacerations, there have been no population comparison studies directed at these important predictive lesions, to our knowledge. The purpose of this study was to determine the comparative frequency of lacerations in AHT versus AI and improve our understanding of the pattern of brain injury in AHT among victims younger than 3 years of age. Lacerations may represent a pattern of injury unique to AHT and are likely the result of angular acceleration, deceleration, and rotational forces that occur when infants have AHT whether through shaking and/or impact or even crushing injuries. With this information, we aim to establish additional imaging features that, in some cases, could be specific for AHT.

\section{MATERIALS AND METHODS \\ Patients}

This study was approved by the University of Utah review board. Retrospective review of the medical records and cross-sectional brain imaging (NCCT and MR imaging) were performed in 2 patient cohorts of infants and children younger than 3 years of age treated between January 2005 and May 2013 at a pediatric hospital verified by the American College of Surgeons as a Level I Pediatric Trauma Center.

For the AHT group, a search of the hospital Child Protective Services data base identified records of 291 patients who either by confession and/or evaluation by the institutional child abuse medical evaluation team were determined to have inflicted trauma. Cases were excluded if brain MR imaging was not performed because MR imaging served to confirm and characterize intracranial injury. Cases were also excluded if no brain pathology was observed on imaging. One case was excluded for suspected meningoencephalitis. Patients who had extensive birth trauma (forceps or failed forceps delivery) were also excluded.

For the AI cohort, a search of the hospital trauma data base identified 214 patients who were coded as having moderate-tosevere traumatic brain injury, as indicated by a presenting Glasgow Coma Scale score of $<13$. Eighty-seven cases were subsequently determined to have been AHT and were excluded from the AI cohort. Of the remaining 127 cases, we further excluded the following: cases of drowning $(n=5)$; underlying vascular or congenital malformation $(n=2)$; and brain imaging not performed or no brain pathology observed on NCCT/MR imaging $(n=92)$.

\section{Imaging}

All imaging was reviewed by a pediatric neuroradiologist and a radiology resident. The reviewers were not blinded to the cohorts. NCCT brain imaging was performed and reviewed for calvarial/ skull base fracture, extra-axial hemorrhage, parenchymal injury, and extracalvarial soft-tissue injury. It was noted whether a laceration was suspected on NCCT by a parenchymal linear focus of high or low attenuation, which was carefully evaluated by subsequent MR imaging.
Brain MR imaging was conducted at $1.5 \mathrm{~T}$ or $3 \mathrm{~T}$ by using commercially available platforms. In most cases, a routine trauma protocol was used, consisting of sagittal T1; axial T1, FLAIR, dual-echo proton density, and T2; coronal gradient-echo imaging (GRE) and FSE T2 sequences. In some cases, postcontrast T1WI axial and coronal sequences and 3D sagittal spoiled gradient-recalled with isotropic axial and coronal reformations were acquired. MR images were reviewed for extra-axial collections and parenchymal injuries, including edema, ischemia, parenchymal hemorrhage/contusion, shear injury, or lacerations. MR imaging was typically performed on the day of or up to 5 days after presentation.

MR imaging was deemed the criterion standard for identifying a parenchymal laceration. The MR imaging criteria for diagnosing a laceration included the following: 1) a parenchymal cleft containing CSF, hemorrhage, or a CSF-hemorrhage fluid level; 2) a linear or oval cleft lined with a paramagnetic substance (ie, ferritin, hemosiderin) demonstrating gradient-echo imaging or SWI hypointensity or a perimeter of diffusion restriction; and 3) a linear signal alteration of $>5 \mathrm{~mm}$ (including T2/FLAIR hyperintense signal and/or T1 shortening) that did not conform to a sulcus or perivascular space.

Skeletal surveys, CT of the abdomen and pelvis, and clinical records were also reviewed to document the presence of non-CNS injuries such as musculoskeletal and solid organ injuries.

\section{Data Analysis}

Tests for association were conducted by using the Fisher exact test, and differences in group distributions were compared by using the Wilcoxon rank sum test. Statistical differences were considered significant if the probability of type 1 error was $<5 \%$. Statistical analyses were performed by using STATA, Version 12.1 (StataCorp, College Station, Texas).

\section{RESULTS}

A total of 137 patients met the eligibility criteria for inclusion into the AHT group, and 28 patients met the criteria for the AI group. The almost 5-fold difference in group size was mainly due to the infrequent use of MR imaging for patients with accidental head injury. Patients of the AHT group were younger than those of the AI group (median, 0.50 versus 1.37 years, respectively; $P<.001$ ) though the sex was similar, as reported in Table 1. Patients within the AI cohort presented to the emergency department with a mean Glasgow Coma Scale score of 5.9 (18 of 28 scores were affected by paralytics).

Lacerations were identified in $18(13.1 \%)$ of the 137 cases of AHT, while none $(0 \%)$ were detected in the patients with AI. This finding represents a $13 \%$ difference in the risk of brain laceration between the groups and may indicate an association between head injury mechanism and laceration $(P=.045)$. These results suggest that the presence of a laceration can indicate an abusive cause of brain injury with a sensitivity and negative predictive value of only $13.1 \%$ and $19.0 \%$, respectively, but with both specificity and positive predictive values of $100 \%$.

Of the AHT cohort found to have lacerations, 10 cases had 
documented emergency department Glasgow Coma Scale scores with an average of 4.5 ( 7 of 10 were affected by paralytics). Eleven of $18(60 \%)$ cases demonstrated physical evidence of impact to the head such as calvarial fractures $(n=9)$ or extracalvarial soft-tissue swelling $(n=2)$. Eleven cases also had imaging evidence of extra-CNS injuries, including solid organ or musculoskeletal injuries. Variably present were findings of other parenchymal injuries, including contusion (20\%), shear injury (40\%), and ischemic injury (40\%). Only 3 cases $(17 \%)$ had no extracranial evidence of head impact and no imaging evidence for extra-CNS injury.

Half (9/18) of the AHT cohort with lacerations demonstrated evidence of retinal hemorrhages on MR imaging. In the AI cohort, only 3 of $28(11 \%)$ patients with accidental trauma had retinal hemorrhages by funduscopic examination.

The exact mechanisms of injury for the AHT cohort are largely unknown. A summary of patient characteristics and mechanisms of injury among the AI group are listed in Table 2.

Table 2: Summary of characteristics of patients with AHT/PBL and $\mathrm{Al}$ and imaging findings

\begin{tabular}{lcc}
\hline & $\begin{array}{c}\text { AHT Cohort with PBLs } \\
(\boldsymbol{n}=\mathbf{1 8})\end{array}$ & $\begin{array}{c}\text { Al Cohort } \\
(\boldsymbol{n}=\mathbf{2 8})\end{array}$ \\
\hline Type of accident & & \\
$\quad$ MVA & & 8 \\
Pedestrian vs auto & & 6 \\
Fall & & 9 \\
Crush & & 2 \\
$\quad$ Horse & 16 & 2 \\
$\quad$ Unknown & 2 & 1 \\
Shaking injury & 9 & \\
Calvarial fracture & 9 & 20 \\
Suture diastasis & & 1 \\
Extra-axial hematoma & 1 & \\
$\quad$ Epidural & 17 & 3 \\
$\quad$ Subdural & 11 & 21 \\
$\quad$ Subarachnoid & 4 & 17 \\
Contusion & 7 & 8 \\
Shear injury & 7 & 14 \\
Ischemic injury & 18 & 4 \\
Laceration & 10 & 0 \\
Extra-CNS injuries & 3 & 10 \\
Deceased & & \\
\hline
\end{tabular}

Note:-PBLs = parenchymal brain lacerations; MVA, motor vehicle accident.
Of the 18 cases of patients with AHT with lacerations, 7 (39\%) demonstrated multiple lacerations. Most lacerations were hemorrhagic linear tears or clefts in the subcortical white matter (SCWM) (Fig 1). Lacerations were present in various regions, with frontal lobes predominating. In half of the cases (9/18), lacerations were suspected on NCCT (generally lesions longer than $1.5 \mathrm{~cm}$ or fluid-filled clefts), whereas the remaining were only identified by MR imaging. MR imaging characteristics include GRE blooming or diffusion restriction along the margins of the tear or a cleft with a fluid-hematocrit level (Figs 2 and 3). A few lacerations were surrounded by vasogenic edema. The On-line Table summarizes imaging characteristics of lacerations and details on which particular MR imaging sequence the lacerations were optimally visualized.

When available, brain imaging remote from presentation was reviewed to evaluate the evolution of the injury and characterize the appearance of chronic lacerations. The most common remote imaging finding in patients with both AHT and AI was resolution of extra-axial blood products and regional or diffuse encephalomalacia or cortical atrophy. Some lacerations healed without obvious residual parenchymal alteration that would meet the criteria for a laceration. Other lacerations of $\geq 2$ months remote from presentation were clearly identified as clefts with central T2 hyperintense signal or a persistent fluid-fluid level or hemosiderin-lined tear (Fig 4).

\section{DISCUSSION}

Imaging plays a crucial role in the characterization of pediatric head trauma. The differentiation of abusive from accidental head injury relies heavily on imaging observations, accumulated experience, and peer-reviewed literature. Our review of AHT and AI cohorts suggests that parenchymal brain lacerations should be considered proxies for AHT.

As early as 1957, Freytag and Lindenberg ${ }^{14}$ described the pathomorphology of cortical cleft contusions (lacerations) and their association with trauma. In 1969, these authors reported postmortem findings in 16 infants with reported trauma $(n=9)$ or postmortem evidence of injury $(n=7) .{ }^{3}$ All had SAH, SDH, and evidence of bodily violence, including bruising. These infants

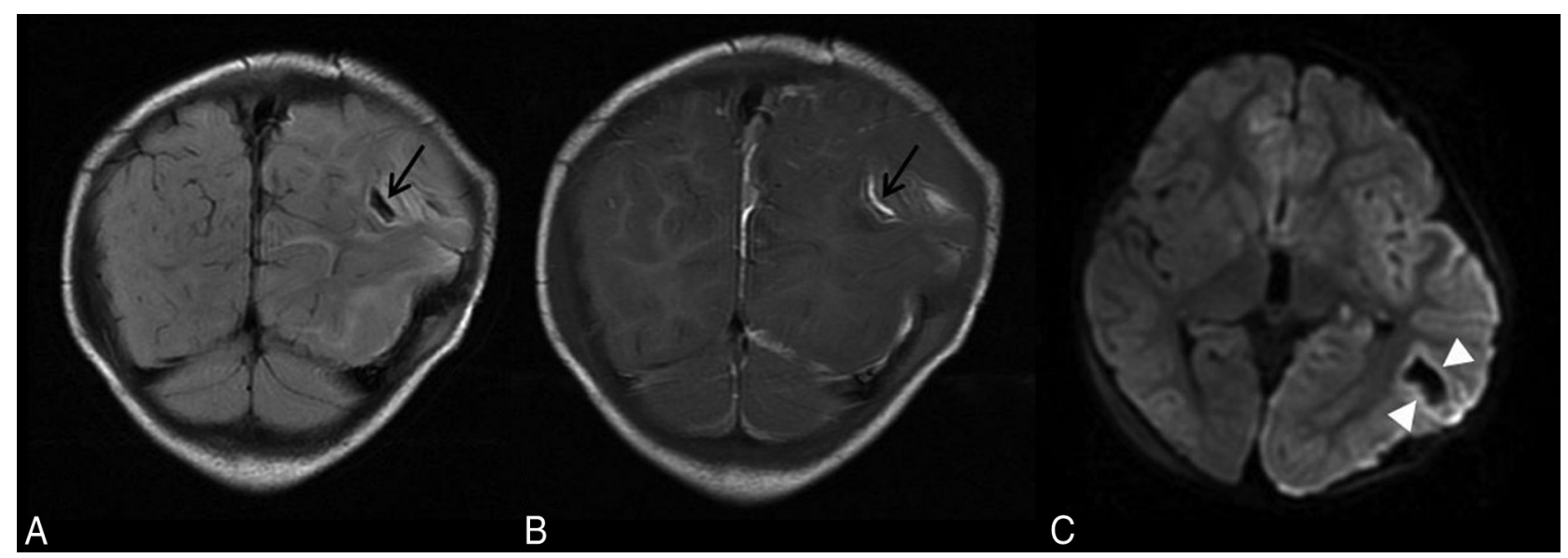

FIG 1. Parenchymal brain lacerations. Case 135. Coronal T2 FLAIR (A) and TIWI (B) demonstrate a curvilinear cleft (black arrows) in the left posterior temporoparietal region containing blood products consistent with acute laceration. $C$, Axial DWI demonstrates marginal diffusion hyperintensity (white arrowheads). 


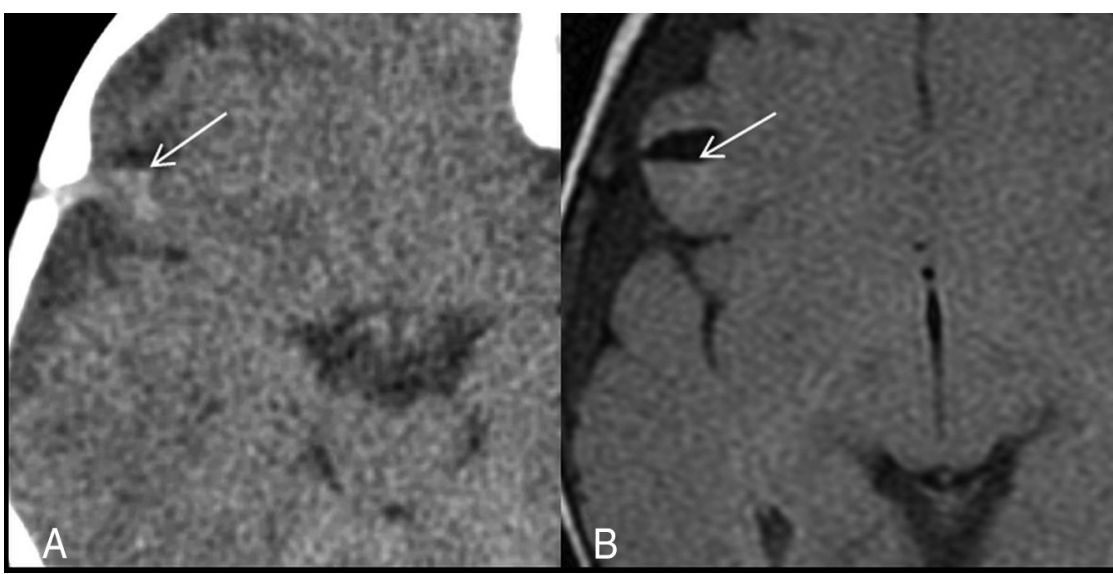

FIG 2. Parenchymal brain laceration with a fluid level. Case 18. A, Axial NCCT image demonstrates an SCWM cleft with a CSF-hemorrhage fluid level. B, Axial T2 FLAIR image depicts a fluid level with layering blood products isointense to gray matter.

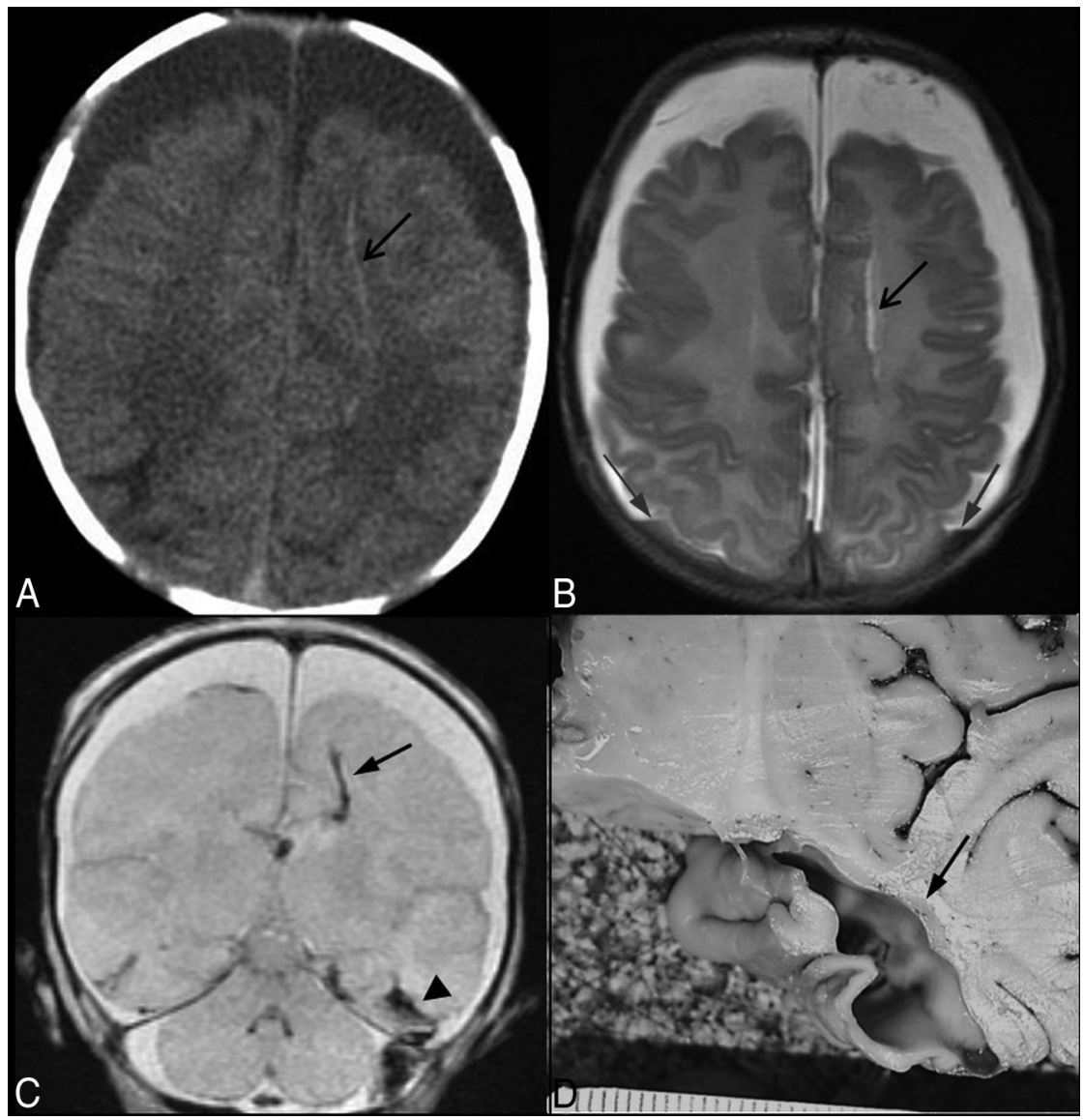

FIG 3. Parenchymal brain lacerations. Case 59. A, Axial NCCT image depicts long linear highattenuation laceration in the left frontal SCWM (black arrow) and bifrontal CSF-attenuation extra-axial collections. B, The corresponding level on an axial T2WI demonstrates a linear parenchymal tear with a fluid signal (black arrow) and hematocrit level within bilateral subdural hemorrhages (gray arrows). C, Coronal GRE image demonstrates blooming from blood products within left frontal (black arrow) and bilateral (black arrowhead) temporal lacerations. A subdural hemorrhage is present above and below the cerebellar tentorium. $D$, A photograph of specimen from brain cutting demonstrates the left temporal SCWM laceration (black arrow).

exhibited macroscopic cerebral hemispheric white matter tears. The white matter tears were described as smooth-walled, with "fresh" tears containing blood and "older" tears demonstrating faint or no blood-product staining. The authors speculated that the gelatin-like consistency of the poorly myelinated infant brain predisposed to parenchymal tearing in the context of shear force generated from trauma.

Calder et $\mathrm{al}^{5}$ reported their results in 12 patients with inflicted head injury. In their study, brains of victims younger than 5 months of age $(n=9)$ showed SCWM tears, while patients older than 5 months of age $(n=3)$ showed more adult-like white matter injury with diffuse axonal injury, axonal retraction balls, and axonal swelling. The authors concluded that the white matter tears represented the manifestation of mechanical damage produced by trauma. They reported that "these clefting lesions of the white matter are important because they represent primary evidence of brain trauma in early infancy." 5

\section{Imaging}

Jaspan et $\mathrm{al}^{13}$ in a series of 6 infants proved to have experienced AHT, reported their cranial sonographic findings of cerebral contusional tears. Frontoparietal and posterior frontal locations of the tears were most common, and SDH was commonly observed. All patients had retinal hemorrhages and fractures, and most had bruising. These authors proposed that the infant's smooth cranial fossae floors, pliable calvaria, patent sutures, and gelatin-like white matter represented the substrate whereby the differential movement of gray matter and white matter in the setting of trauma led to clefting white matter tears. These authors reinforced the earlier observations by Calder et $\mathrm{al}^{5}$ who, a decade earlier, recognized these tears as a proxy for brain trauma in young infants.

Our data recapitulate the pathomorphologic observations of Lindenberg et $\mathrm{al},{ }^{3}$ including the association of lacerations with evidence of AHT, such as SDH and SAH. All cases with lacerations demonstrated extra-axial blood products: Epidural hematoma $(n=1), \mathrm{SDH}$ alone $(n=6)$, and both SAH and a subdural collection such as acute $\mathrm{SDH}$ and/or chronic SDH/hygroma $(n=11)$. Seven cases demonstrated purely acute SDH, while 2 cases had evidence of growing traumatic hygromas. Eight of the 17 cases with SDH \pm SAH demonstrated mixed-attenuation collections. 


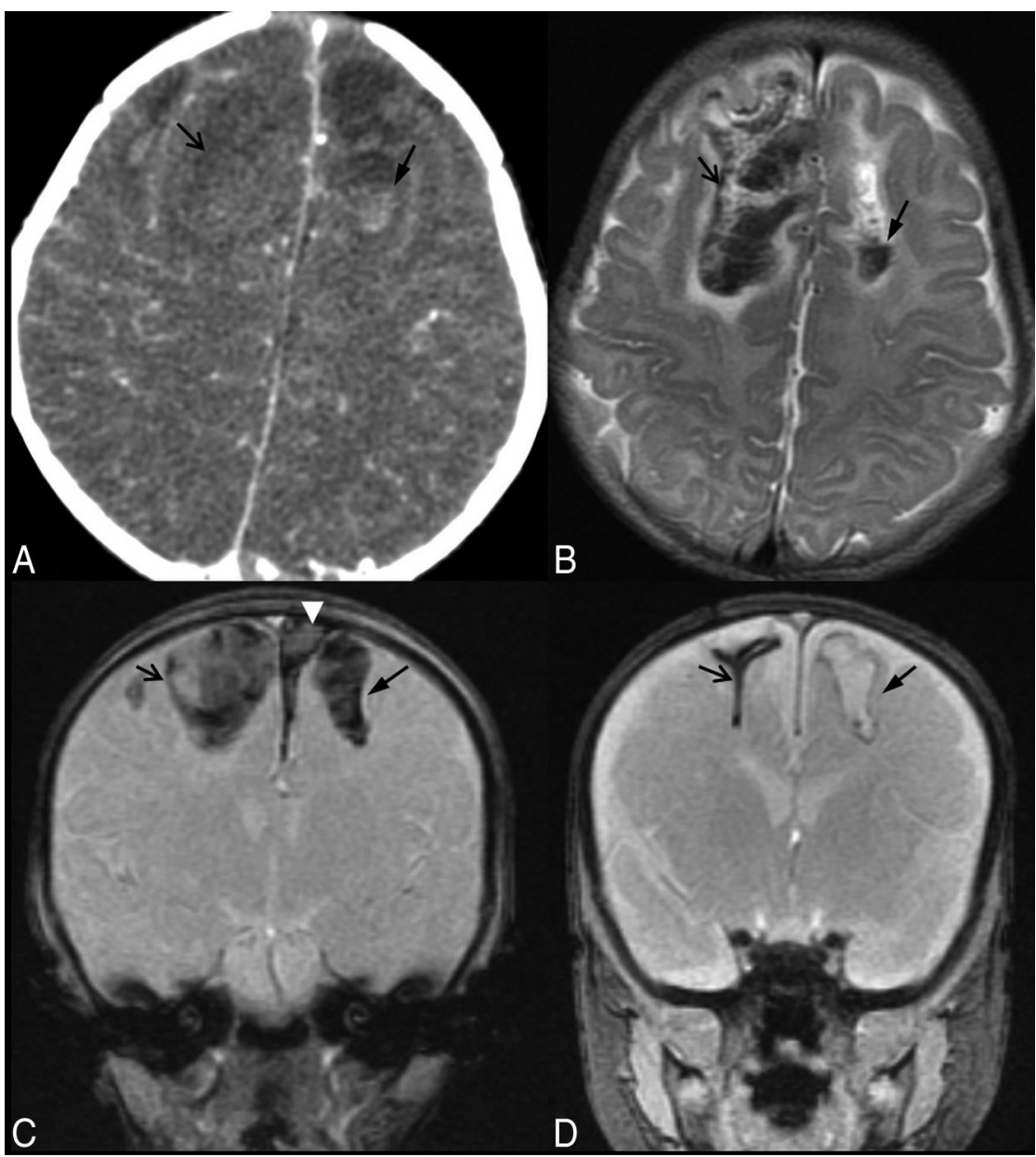

FIG 4. Parenchymal brain lacerations. Case 53. A, Axial NCCT image depicts a bifrontal parenchymal clefting injury with a hematocrit level within the left frontal laceration. $B$, The corresponding level on an axial T2WI better demonstrates the acute hemorrhagic SCWM parenchymal clefts with a fluid-hematocrit level and mild surrounding edema. C, Coronal GRE image 1 day after the acute injury demonstrates blooming artifacts of the blood-filled wide parenchymal clefts in the SCWM of the frontal lobes. Note the left parasagittal SDH (white arrowhead). D, Coronal GRE image 2 months following injury demonstrates blooming from hemosiderin-lined clefts (arrows). There has been marked retraction of the blood clot and near-apposition of the walls of the cleft within the right frontal lobe parenchymal brain laceration, while the cleft in the left SCWM remains wide and fluid-filled.

In 1 case, the collection was surgically evacuated and found to be consistent with acute hemorrhage; 3 cases had evidence of loculated clots or membranes suggestive of acute or chronic SDH; and 4 had low-attenuation collections that could have represented either hygroma or chronic SDH.

In our study, there were 2 laceration cases with perpetrator confession of shaking injury, both of which also had signs of impact. Evidence of impact, such as calvarial fracture or extra-calvarial soft-tissue swelling, was not always present in the cases with lacerations. Of the AHT cases with lacerations, a minimum of 9 (50\%) cases had retinal hemorrhages, and 7 (39\%) cases lacked signs of impact to the head, suggesting that shaking injury could result in parenchymal laceration.

During the study period, we incidentally encountered head NCCT imaging of a patient presenting for a reason other than acute trauma, which demonstrated findings of chronic bifrontal lacerations. This finding emphasizes that radiologists should be aware of encountering lacerations remote from trauma, such as in a child pre- senting with seizures or developmental delay without a history of reported trauma. Thus, the interpreting radiologist should appreciate the finding of a parenchymal brain laceration as a proxy for AHT.

\section{Differential Diagnosis}

In the setting of suspected pediatric head trauma, a review of the health history, appropriate laboratory testing, and cross-sectional brain imaging allow the child abuse pediatrician to develop a focused differential diagnosis. Despite clinical, imaging, and forensic evidence of AHT as a cause of parenchymal brain laceration, there are individuals who posit that such lacerations found in neonates and young infants lack diagnostic specificity.

An association between instrumented delivery and neurologic injury is well-recognized. Au-Yong et $\mathrm{al}^{15}$ reported 5 children with cerebral cortical tears, 4 of which had a history of difficult deliveries (4 forceps, 1 episode of antepartum hemorrhage). A combination of ultrasound, NCCT, and MR imaging was used in the investigation of neurologic abnormality (seizures, hypertonia, eye deviation, and increasing head circumference). All patients were studied with MR imaging. Most interesting, MR imaging in these 5 patients depicted clefting lesions extending through the cerebral cortex. ${ }^{15}$ This finding differs from the subcortical clefting reported from postmortem series of injured infants and the imaging manifestations of AHT-associated brain parenchymal clefts, including our data showing parenchymal brain lacerations only in the AHT cohort. ${ }^{1,2,11-13,16}$

Multicystic encephalomalacia reflects the underlying subcortical necrosis of white matter and diffuse loss of the cerebral cortical neurons. It is most commonly observed following a perinatal hypoxic-ischemic injury. Less commonly, multicystic encephalomalacia may follow AHT. Other intracranial injuries (SAH, $\mathrm{SDH}$ ) are often detected in the acute and subacute periods following AHT. The imaging characteristics of multicystic encephalomalacia, including diffuse cerebral cortical atrophy and multiple subcortical cysts, are not likely to be confused with the parenchymal brain lacerations seen in AHT. ${ }^{17}$

The cystic and cavitary necrotic white matter findings of late neonatal and early infantile CNS infections (agents such as congenital cytomegalovirus infections, Parechovirus encephalitis, and Citrobacter meningoencephalitis) are characterized by their distinctive clinical presentations, CSF chemistries, microbiologic assays, polymerase chain reaction results, and imaging character- 
istics, which bear no resemblance to the lacerations that have been reported among victims of AHT. ${ }^{18}$

\section{Limitations}

Because this was a retrospective study conducted at a single institution, certain inherent limitations may apply, such as generalizability and bias in patient selection. In addition, interpretations of images were not conducted in a blinded fashion with respect to study cohorts.

Setting a high standard for imaging evaluation by using MR imaging to determine the presence of lacerations limited the size of our cohorts. This limitation was especially true for the AI cohort because serial neurologic examinations and negative NCCT results did not warrant brain MR imaging. MR imaging would be even less frequent for cases of mild traumatic brain injury.

Although the age range of both groups was similar, the median age of patients in the AHT cohort was younger than those in the AI cohort; this difference suggests that age-related brain maturation, along with the mechanism of injury, may play a role in the prevalence of lacerations between the 2 cohorts. Sixteen of 18 patients with laceration were younger than 1 year. However, 8 of 28 patients with AI were also younger than 1 year of age. It has been postulated that lacerations only occur in the youngest patients, when brain substance is more gelatinous, due to lack of mature myelination. Brain turgor has been estimated to mature by 2-3 years of age (personal communication by phone, L.B. Rorke-Adams, MD, August 2, 2013). The oldest patient with a laceration was 29 months, suggesting that lacerations are not exclusive to the immature brain substance of infants. Further studies with larger sample sizes, particularly of very young patients, are warranted to more thoroughly address this potential confounder.

\section{CONCLUSIONS}

In our observational review of young pediatric head trauma groups (AHT and AI), brain parenchymal lacerations were only identified in the AHT cohort. When present, lacerations were always associated with other findings of CNS trauma, including retinal hemorrhages and SDHs with SAH. The median age in the AHT cohort was substantially younger than that in the AI cohort; this finding confirms prior reports that child abuse is most common in the first year of life.

Only half of the lacerations characterized by MR imaging were visualized by NCCT, emphasizing the importance of MR imaging for the detection of parenchymal injury. Even with a normal head NCCT examination, and especially in a child younger than 1 year of age, we strongly encourage MR imaging to evaluate for parenchymal injury and extra-axial hemorrhage, which are important diagnostic and prognostic considerations. Hemosiderin-lined or fluid-filled brain parenchymal clefts or linear blooming (SWI and GRE) parenchymal abnormalities observed in an infant or young child evaluated for reasons other than acute trauma should raise concern for prior inflicted brain injury.
Disclosures: Lori D. Frasier-UNRELATED: Expert Testimony: compensated legal consultation and testimony in various jurisdictions in the United States. Consultancy: legal consultation without testimony in various jurisdictions in the United States; Children's Healthcare of Minnesota (peer-review and quality improvement consultant). Honoraria: for invited lectures, including service on Speaker's bureaus. Gary L. Hedlund-UNRELATED: Expert Testimony: compensated legal consultation and testimony in various jurisdictions in the United States. Honoraria: for invited lectures.

\section{REFERENCES}

1. Jenny C, Hymel KP, Ritzen A, et al. Analysis of missed cases of abusive head trauma. JAMA 1999;281:621-26 CrossRef Medline

2. Hedlund GL, Frasier LD. Neuroimaging of abusive head trauma. Forensic Sci Med Pathol 2009;5:280-90 CrossRef Medline

3. Lindenberg R, Freytag E. Morphology of brain lesions from blunt trauma in early infancy. Arch Pathol 1969;87:298-305 Medline

4. Ordia IJ, Strand R, Gilles F, et al. Computerized tomography of contusional clefts in the white matter of infants: report of two cases. J Neurosurg 1981;54:696-98 CrossRef Medline

5. Calder IM, Hill I, Scholtz CL. Primary brain trauma in non-accidental injury. J Clin Pathol 1984;37:1095-1100 CrossRef Medline

6. Hausdorf G, Helmke K. Sonographic demonstration of contusional white matter clefts in an infant. Neuropediatrics 1984;15:110-12 CrossRef Medline

7. Sato Y, Yuh WT, Smith WL, et al. Head injury in child abuse: evaluation with MR imaging. Radiology 1989;173:653-57 CrossRef Medline

8. Kleinman PK. Diagnostic Imaging of Child Abuse. 2nd ed. St Louis: Mosby-Year Book; 1998:315-18

9. Tung GA, Kumar M, Richardson RC, et al. Comparison of accidental and nonaccidental traumatic head injury in children on noncontrast computed tomography. Pediatrics 2006;118:626-33 CrossRef Medline

10. Bechtel K, Stoessel K, Leventhal JM, et al. Characteristics that distinguish accidental from abusive injury in hospitalized young children with head trauma. Pediatrics 2004;114:165-68 CrossRef Medline

11. Ewing-Cobbs L, Prasad M, Kramer L, et al. Acute neuroradiologic findings in young children with inflicted or noninflicted traumatic brain injury. Childs Nerv Syst 2000;16:25-33; discussion 34 CrossRef Medline

12. Kemp AM, Jaspan T, Griffiths J, et al. Neuroimaging: what neuroradiological features distinguish abusive from non-abusive head trauma? A systematic review. Arch Dis Child 2011;96:1103-12 CrossRef Medline

13. Jaspan T, Narborough G, Punt JA, et al. Cerebral contusional tears as a marker of a child abuse: detection by cranial sonography. Pediatr Radiol 1992;22:237-45 CrossRef Medline

14. Freytag E, Lindenberg R. Morphology of cortical contusions. AMA Arch Pathol 1957;63:23-42 Medline

15. Au-Yong IT, Wardle SP, McConachie NS, et al. Isolated cerebral cortical tears in children: aetiology, characterisation and differentiation from non-accidental head injury. Br J Radiol 2009;82:735-41 CrossRef Medline

16. Duhaime AC, Gennarelli TA, Thibault LE, et al. The shaken baby syndrome: a clinical, pathological, and biomechanical study. J Neurosurg 1987;66:409-15 CrossRef Medline

17. Matlung SE, Bilo RA, Kubat B, et al. Multicystic encephalomalacia as an end-stage finding in abusive head trauma. Forensic Sci Med Pathol 2011;7:355-63 CrossRef Medline

18. Hedlund G, Bale JF, Barkovich AJ. Infections of the developing and mature nervous system. In: Barkovich AJ, Raybaud C, eds. Pediatric Neuroimaging. 5th ed. Philadelphia: Lippincott Williams \& Wilkins; 2012:954-1050 